\title{
Experimental evidence for lamellar magnetism in hemo-ilmenite by polarized neutron scattering
}

Brok, Erik; Sales, Morten; Lefmann, Kim; Kuhn, Luise Theil; Schmidt, Wolfgang F.; Roessli, Bertrand; Robinson, Peter; McEnroe, Suzanne A.; Harrison, Richard J.

Published in:

Physical Review B Condensed Matter

Link to article, DOI:

10.1103/PhysRevB.89.054430

Publication date:

2014

Document Version

Publisher's PDF, also known as Version of record

Link back to DTU Orbit

Citation (APA):

Brok, E., Sales, M., Lefmann, K., Kuhn, L. T., Schmidt, W. F., Roessli, B., Robinson, P., McEnroe, S. A., \& Harrison, R. J. (2014). Experimental evidence for lamellar magnetism in hemo-ilmenite by polarized neutron scattering. Physical Review B Condensed Matter, 89(5), [054430]. https://doi.org/10.1103/PhysRevB.89.054430

\section{General rights}

Copyright and moral rights for the publications made accessible in the public portal are retained by the authors and/or other copyright owners and it is a condition of accessing publications that users recognise and abide by the legal requirements associated with these rights.

- Users may download and print one copy of any publication from the public portal for the purpose of private study or research.

- You may not further distribute the material or use it for any profit-making activity or commercial gain

- You may freely distribute the URL identifying the publication in the public portal 


\title{
Experimental evidence for lamellar magnetism in hemo-ilmenite by polarized neutron scattering
}

\author{
Erik Brok,,${ }^{1,2}$ Morten Sales, ${ }^{3,4}$ Kim Lefmann, ${ }^{3}$ Luise Theil Kuhn,,${ }^{5}$ Wolfgang F. Schmidt, ${ }^{6}$ Bertrand Roessli, ${ }^{7}$ \\ Peter Robinson, ${ }^{8}$ Suzanne A. McEnroe, ${ }^{9}$ and Richard J. Harrison ${ }^{10,}{ }^{,}$ \\ ${ }^{1}$ Department of Physics, Technical University of Denmark, DK-2800 Kgs Lyngby, Denmark \\ ${ }^{2}$ Center for Electron Nanoscopy, Technical University of Denmark, DK-2800 Kgs Lyngby, Denmark \\ ${ }^{3}$ Nano-Science Center, Niels Bohr Institute, University of Copenhagen, DK-2100 Copenhagen Ø, Denmark \\ ${ }^{4}$ Department for Methods for Characterization of Transport Phenomena in Energy Materials, \\ Helmholtz-Zentrum Berlin Für Materialen und Energie GmbH, D-14109 Berlin, Germany \\ ${ }^{5}$ Department of Energy Conversion and Storage, Technical University of Denmark, DK-4000 Roskilde, Denmark \\ ${ }^{6} J$ ülich Centre for Neutron Science JCNS, Forschungszentrum Jülich GmbH, Outstation at ILL, 38042 Grenoble, France \\ ${ }^{7}$ Laboratory for Neutron Scattering, Paul Scherrer Institute, 5232 Villigen, Switzerland \\ ${ }^{8}$ Geological Survey of Norway, N-7491 Trondheim, Norway \\ ${ }^{9}$ Norwegian University of Science and Technology, N-7491 Trondheim, Norway \\ ${ }^{10}$ Department of Earth Sciences, University of Cambridge, CB2 3EQ, United Kingdom
}

(Received 13 November 2013; published 26 February 2014)

\begin{abstract}
Large local anomalies in the Earth's magnetic field have been observed in Norway, Sweden, and Canada. These anomalies have been attributed to the unusual magnetic properties of naturally occurring hemo-ilmenite, consisting of a paramagnetic ilmenite host $\left(\alpha-\mathrm{Fe}_{2} \mathrm{O}_{3}\right.$-bearing $\left.\mathrm{FeTiO}_{3}\right)$ with exsolution lamellae $(\approx 3 \mu \mathrm{m}$ thick $)$ of canted antiferromagnetic hematite $\left(\mathrm{FeTiO}_{3}\right.$-bearing $\left.\alpha-\mathrm{Fe}_{2} \mathrm{O}_{3}\right)$ and the mutual exsolutions of the same phases on the micron to nanometer scale. The origin of stable natural remanent magnetization (NRM) in this system has been proposed to be uncompensated magnetic moments in the contact layers between the exsolution lamellae. This lamellar magnetism hypothesis is tested here by using polarized neutron diffraction to measure the orientation of hematite spins as a function of an applied magnetic field in a natural single crystal of hemo-ilmenite from South Rogaland, Norway. Polarized neutron diffraction clearly shows that the ilmenite spins do not contribute to the NRM and that hematite spins account for the full magnetization at ambient temperature. Hematite sublattice spins are shown to adopt an average angle of $56^{\circ}$ with respect to a saturating magnetic field, which is intermediate between the angle of $90^{\circ}$ predicted for a pure canted moment and the angle of $0^{\circ}$ predicted for a pure lamellar moment. The observed NRM is consistent with the vector sum of lamellar magnetism and canted antiferromagnetic contributions. The relative importance of the two contributions varies with the length scale of the microstructure, with the lamellar contribution increasing when exsolution occurs predominantly at the nanometer rather than the micrometer scale.
\end{abstract}

DOI: 10.1103/PhysRevB.89.054430

PACS number(s): 75.25.-j, 91.60.Pn, 75.70.Cn, 75.75.-c

\section{INTRODUCTION}

The mineral intergrowth hemo-ilmenite consists of an ilmenite host $\left(\mathrm{FeTiO}_{3}\right)$ with several populations of hematite $\left(\alpha-\mathrm{Fe}_{2} \mathrm{O}_{3}\right)$ exsolution lamellae. Hemo-ilmenite has been studied extensively because of its importance as a source of anomalies in the magnetic field of the Earth $[1,2]$ and potentially also on Mars [3], and because of its unusual magnetic properties that are not explained by the magnetic properties of the individual constituent minerals. Natural samples of hemo-ilmenite have a large and extremely stable natural remanent magnetization, which is believed to be related to the fine exsolution structure of the intergrown hematite and ilmenite phases $[2,4]$. Solid solution hematite-ilmenite $\left[x \mathrm{FeTiO}_{3}-(1-x) \mathrm{Fe}_{2} \mathrm{O}_{3}\right]$ with compositions in the range $0.5<$ $x<0.85$ are magnetic semiconductors [5,6] and a detailed understanding of the complex magnetic properties of natural samples of nanostructured hemo-ilmenite could lead to important discoveries that have application potential in spintronics devices [7-9]. Hemo-ilmenite consists of the minerals ilmenite $\left(\mathrm{FeTiO}_{3}\right)$ which is paramagnetic at room temperature, but is

\footnotetext{
*rjh40@esc.cam.ac.uk
}

AFM ordered below a Néel temperature of about $58 \mathrm{~K}[10]$, and hematite $\left(\alpha-\mathrm{Fe}_{2} \mathrm{O}_{3}\right)$ which is antiferromagnetic (AFM) with a Néel temperature of $955 \mathrm{~K}$ [11]. The quoted Néel temperatures are for the pure minerals (end members) and the Néel temperatures of the hematite and ilmenite phases of our natural sample are expected to be lowered because of cation substitution.

Hematite crystallizes in the $R \overline{3} c$ (corundum) structure with the $\mathrm{Fe}^{3+}$ magnetic moments ferromagnetically aligned within the basal $(a b)$ planes of the hexagonal structure, while moments on adjacent planes are antiparallel apart from a small canting of approximately $0.065^{\circ}$ [11]. The canting, which is in the basal plane, gives a small net magnetic moment, and hematite is thus often referred to as a weak ferromagnet, rather than an antiferromagnet. There are three easy axes in the basal plane giving six possible antiferromagnetic domains. In all of these domains the spins are in the basal plane and nearly perpendicular to one of the hexagonal $a$ axes. In pure bulk hematite the spins undergo the so-called Morin transition at $\left(T_{\mathrm{M}} \approx 264 \mathrm{~K}[12,13]\right)$. The Morin transition is a spin-flop transition, where the two AFM sublattices change their spin direction from perpendicular to parallel to the $c$ axis. However, substitution of even small amounts $(\approx 1 \%)$ of Ti in hematite is known to suppress the transition $[12,13]$ and it does not occur 

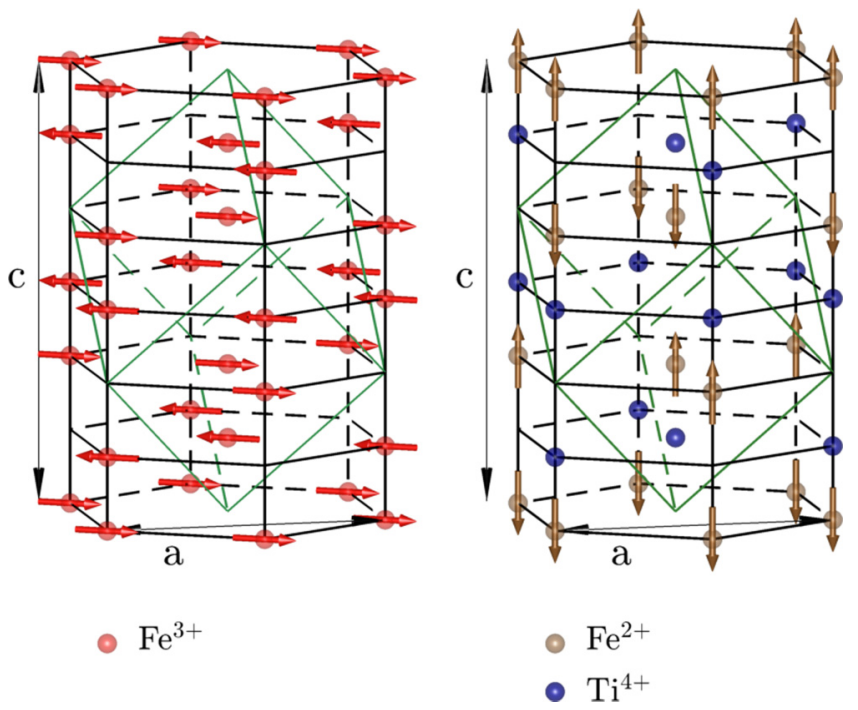

FIG. 1. (Color online) Antiferromagnetic structure of hematite and ilmenite. Left: Magnetic structure of hematite above the Morin transition. Right: Magnetic structure of ilmenite. The oxygen atoms are left out of the drawings, as is the small canting of the $\mathrm{Fe}^{3+}$ moments in hematite.

in hemo-ilmenite samples. The crystal structure of ilmenite is $R \overline{3}$ and is identical to the hematite structure, but with alternating layers of $\mathrm{Fe}^{2+}$ and $\mathrm{Ti}^{4+}$ ions instead of $\mathrm{Fe}^{3+}$. In ilmenite below the Néel temperature the $\mathrm{Fe}^{2+}$ moments are aligned along the $c$ axis and antiparallel between adjacent $\mathrm{Fe}^{2+}$ layers (see Fig. 1). The lattice parameters of hematite and ilmenite are very similar $(a=b=5.038 \AA$ and $c=13.772$ $\AA$ for hematite [11], and $a=b=5.088 \AA$ and $c=14.085$ $\AA$ for ilmenite $[14,15])$ and the two phases are thus able to grow epitaxially together. In natural samples of hemo-ilmenite that slowly cooled around a billion years ago [16], the two phases exhibit a complex exsolution structure that has been investigated with electron microscopy [17], revealing multiple generations of epitaxially aligned intergrown lamellae ranging in thickness from several microns to a few nanometers in the direction of the crystallographic $c$ axis. The lamellae are flattened in the $c$ direction and extended in the basal plane. While the AFM sublattice direction in hematite above the Morin temperature is usually assumed to be within the basal plane, a significant out-of-plane angle of about $30^{\circ}$ has been observed in a natural ilmeno-hematite sample showing nanoscale exsolution structure [18].

Natural samples of hemo-ilmenite show a large natural remanent magnetization of around (1.4-9.1) $\times 10^{-3} \mathrm{~A} \mathrm{~m}^{2} / \mathrm{kg}$ [19] that cannot be explained by the ferromagnetic contribution from the canted antiferromagnetic (CAF) hematite. The coercivity of the samples as well as the demagnetization temperature [4] is comparable to that of Ti-substituted hematite. The material is not only strongly magnetic, but the magnetism is also very stable. Uncompensated spins in contact layers between hematite lamellae and the ilmenite host, with magnetization aligned by the geomagnetic field at the time the sample exsolved [4,20-22] have been proposed as an explanation for the strong remanent magnetization. This hypothesis, which directly links the nanoscale exsolution

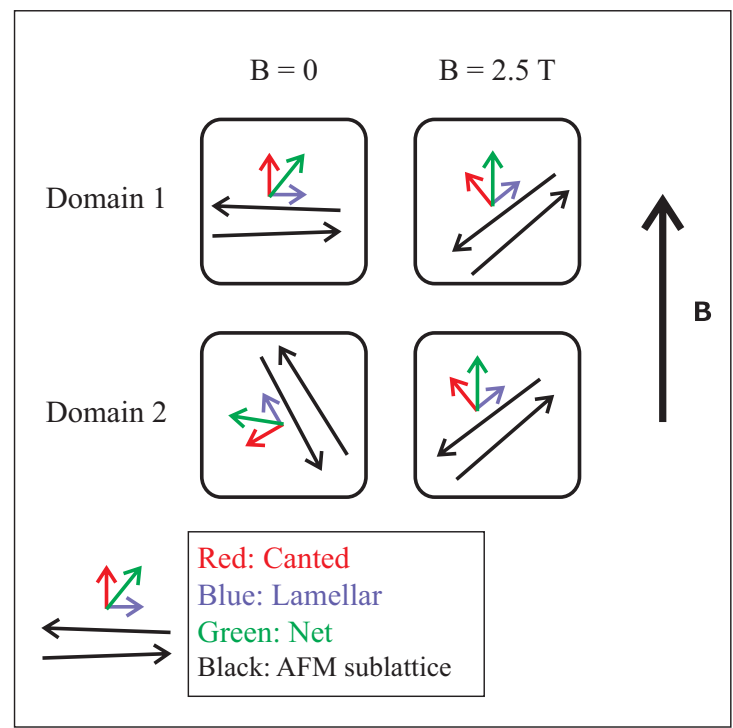

FIG. 2. (Color online) Sketch of the in-plane hematite spin directions and their response to a magnetic field applied applied in the plane. The net moment is the vector sum of the CAF moment, which is almost perpendicular to the AFM sublattice, and the lamellar moment which is parallel to the AFM sublattice. The two "domains" represent two of the possible six antiferromagnetic domains. In zero field the spin orientation depends on the remanent magnetization of the sample. If all six domains are equally represented, or if the spins are randomly oriented, the average spin angle with respect to $\mathbf{B}$ will be $45^{\circ}$. At a saturating field (2.5 $\mathrm{T}$ at room temperature) the net moment is aligned with the field and the average spin orientation is no longer random, but makes an angle with $\mathbf{B}$ that depends on the proportion of canted and lamellar moments.

structure of the hemo-ilmenite samples to their unusual magnetic properties is termed lamellar magnetism and has been backed by Monte Carlo simulations of the cation ordering during exsolution $[4,20]$, and measurement of exchange bias below $T_{\mathrm{N}}$ of ilmenite have confirmed that the NRM is associated with magnetic moments at the lamellar interfaces $[23,24]$.

The response of the magnetic moments to an applied field can give information about the configuration of the atomic spins. In particular, the response of the hematite spins to a saturating field applied in the basal plane can determine whether or not the lamellar magnetism hypothesis is a plausible explanation for the spin structure in the lamellar system. A sketch of the response of the in-plane magnetic moment to a magnetic field applied in the plane is given in Fig. 2.

Here we use the technique of polarized neutron diffraction to examine a natural hemo-ilmenite sample with an exsolution microstructure showing a range of lamellar sizes from coarse to fine. Through uniaxial polarization analysis we determine the average hematite spin direction in the basal plane as a function of applied magnetic fields up to $2.5 \mathrm{~T}$ to directly measure the response of the lamellar moments in order to confirm the validity of the lamellar magnetism hypothesis.

We find that the hematite magnetic moments saturate at an average angle of $56^{\circ}$ to the applied field, which is consistent with lamellar magnetism as an important mechanism for the NRM. 


\section{EXPERIMENTAL DETAILS}

\section{A. Sample characterization}

The sample is a rectangular solid piece, approximately $12 \times 8 \times 8 \mathrm{~mm}^{3}$, with a mass of $1.977 \mathrm{~g}$, cut from a sample of a hemo-ilmenite dike at South Rogaland, Norway. The sample, labeled Pramsknuten 5-1 T, was selected from a larger number of similar pieces by electron backscatter diffraction (EBSD) [19]. The EBSD investigations revealed that the sample is a single crystal of hemo-ilmenite, and established the crystallographic axes with respect to the faces of the sample. $\mathrm{X}$-ray fluorescence (XRF) revealed that the sample is (in percentages of end members) $16.18 \%$ hematite composition and $83.82 \%$ ilmenite composition $[19,25]$. The ilmenite part of the sample contains $19.2 \%$ of the $\mathrm{MgTiO}_{3}$ end member as well as small amounts (less than $1 \%$ ) of $\mathrm{MnTiO}_{3}, \mathrm{ZnTiO}_{3}$, and $\mathrm{NiTiO}_{3}$. In the hematite part of the sample only small substitutions of $\mathrm{Al}_{2} \mathrm{O}_{3}, \mathrm{Cr}_{2} \mathrm{O}_{3}$, and $\mathrm{V}_{2} \mathrm{O}_{3}$ were found (less than $1 \%$ of end members). Importantly no ferromagnetic impurity phases were detected. The NRM of the sample was measured to $2.613 \times 10^{-3} \mathrm{~A} \mathrm{~m}^{2} / \mathrm{kg}$ and found to be oriented close to the basal plane $\left(6.1^{\circ}\right.$ out of plane $)$ and close to one of the basal plane crystallographic axes $\left(6.8^{\circ}\right.$ in-plane angle with nearest hexagonal axis) [19]. The saturation magnetization of the sample is about $0.43 \mathrm{~A} \mathrm{~m}^{2} / \mathrm{kg}$ and the coercivity approximately $60 \mathrm{mT}$ (estimated from magnetization measurements on other pieces of the same rock slab). The saturation magnetization of CAF hematite is $0.404 \mathrm{~A} \mathrm{~m}^{2} / \mathrm{kg}$ [11] and with a mass fraction of $16.88 \%$ hematite (assuming $16.18 \%$ pure hematite and $83.82 \%$ pure ilmenite in the sample) this can at most amount to a magnetization of the sample of $0.0682 \mathrm{~A} \mathrm{~m}^{2} / \mathrm{kg}$. Thus, only about $16 \%$ of the saturation magnetization can be explained by the weak ferromagnetism of hematite.

\section{B. Neutron scattering experiments}

The orientation of the ilmenite and hematite spins was studied by polarized neutron diffraction at the three-axis spectrometer IN12 at Institute Laue-Langevin (ILL), Grenoble, France. We used an initial neutron wavelength of $4.05 \AA$, selected by a PG (002) monochromator. The beam was polarized by a supermirror bender after the monochromator. The analyzer consists of Heusler (111) crystals, selecting one spin state, and oriented to elastic scattering. To improve the $q$ resolution of the instrument, we collimated the beam, using the sequence guide-open-PG-40'-bender-sample-40'-Heusler60 '-detector. A vertical guide field of 2 to $3 \mathrm{mT}$ was applied along the beam path to prevent neutron depolarization. A Mezei-type spin flipper coil was inserted in the final beam path to allow for $180^{\circ}$ rotation of the beam polarization. The flipping ratio of the setup was measured to $R \approx 40$. The sample was aligned with the $\mathbf{a}^{*}$ and $\mathbf{c}^{*}$ axes in the scattering plane and was placed in a cryomagnet, capable of applying a $\pm 2.5 \mathrm{~T}$ vertical field. The sample was oriented by the nuclear ilmenite (003) and hematite $(10 \overline{2})$ reflections.

A preliminary polarized neutron diffraction experiment was performed at the triple-axis spectrometer TASP at PSI, Villigen, Switzerland [26]. The data from this experiment are in general agreement with the data presented here [27], but

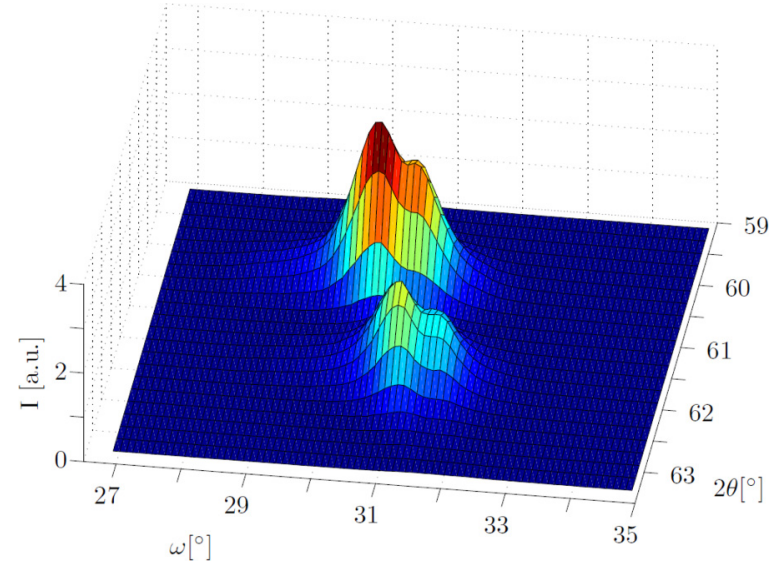

FIG. 3. (Color online) Mapping of the (003) peaks measured at MORPHEUS. $2 \theta$ is the scattering angle and $\omega$ is the azimuthal rotation angle of the sample. The most intense peak at $2 \theta \approx 60^{\circ}$ is the structural ilmenite reflection and the less intense peak at $2 \theta \approx 62^{\circ}$ is the magnetic hematite reflection. Both peaks have a shoulder, indicating that the sample consists of two distinct crystallites oriented at an angle of approximately $0.6^{\circ}$ with respect to each other.

due to the low flipping ratio $(R \approx 4)$, at the used wavelength of $\lambda=4.05 \AA$, we here present only the ILL data.

An additional high-field neutron experiment was performed at the RITA-2 triple-axis spectrometer at PSI [28]. Here the hematite (101) reflection was studied by unpolarized diffraction with the same sample orientation, but using a stronger cryomagnet, capable of applying a $15 \mathrm{~T}$ field.

The crystal structure of the sample was studied with high resolution unpolarized neutron diffraction at the two-axis spectrometer MORPHEUS at PSI. Here we used an incoming wavelength of $4.72 \AA$ and tight collimations: guide-open-PG20'-sample-30'-detector.

\section{RESULTS}

Before presenting the results of the polarization analysis experiment we show the results of the experiment on MORPHEUS, which establishes the crystalline quality of the sample. Figure 3 shows a mapping of the structural (003) ilmenite peak and magnetic (003) hematite peak, measured using unpolarized neutrons in the high resolution experiment at MORPHEUS. The peak "shoulders" reveal that the crystal consists of two crystallites that are oriented at an angle of about $0.6^{\circ}$ with respect to each other. For the purpose of the investigations presented here this mosaicity of $0.6^{\circ}$ is small enough that we consider the sample to be a single crystal.

In the uniaxial polarization analysis experiment performed at IN12 we measured the magnetic (003) hematite reflection to determine the in-plane spin direction. The experimental geometry was as sketched in Fig. 4. Magnetic moments parallel to the incident polarization $\mathbf{P}_{i}$ will only give rise to non spin flip (NSF) scattering, and the NSF cross section is [29]

$$
\sigma^{\mathrm{NSF}}=K M_{\perp z}^{2}
$$

where $K$ is a constant, and $M_{\perp z}$ is the $z$ component of $\mathbf{M}_{\perp}$ parallel to $\mathbf{P}_{i}$, as defined in Fig. 4. $M_{\perp}(\mathbf{q})$ is the Fourier transform of the magnetic moment density perpendicular to 


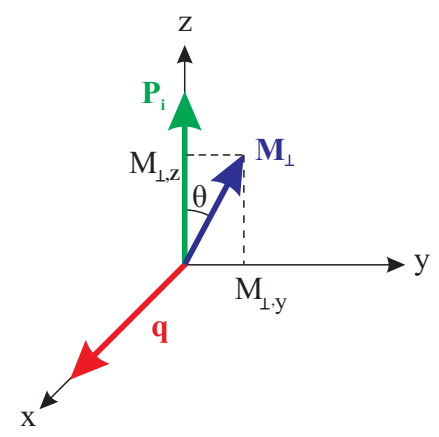

FIG. 4. (Color online) Geometry in the IN12 experiment. $\mathbf{P}_{i}$ is the incoming polarization vector, $\mathbf{q}$ is the scattering vector, and $\mathbf{M}_{\perp}$ is the magnetic sublattice magnetization perpendicular to $\mathbf{q}$. The magnetic field is applied in the $z$ direction (parallel to $\mathbf{P}_{i}$ ). The angle between $\mathbf{M}_{\perp}$ and $\mathbf{P}_{i}$ is called $\theta$.

$\mathbf{q}$, sometimes referred to as the magnetic interaction vector. Moments perpendicular to $\mathbf{P}_{i}$ will only give rise to spin flip (SF) scattering, and the SF cross section is

$$
\sigma^{\mathrm{SF}}=K M_{\perp y}^{2},
$$

where $K$ is the same constant as in (1), and $M_{\perp y}$ is the component of $\mathbf{M}_{\perp}$ perpendicular to $\mathbf{P}_{i}$. From the ratio of the SF to the NSF cross section we can calculate $\theta$ - the angle between $\mathbf{P}_{i}$ and $\mathbf{M}_{\perp}$,

$$
\frac{\sigma^{\mathrm{SF}}}{\sigma^{\mathrm{NSF}}}=\frac{M_{\perp}^{2} \cos ^{2} \theta}{M_{\perp}^{2} \sin ^{2} \theta}=\tan ^{2} \theta .
$$

With the external field applied in the $z$ direction (along $\mathbf{P}_{i}$ ), $\theta$ is the in-plane spin angle with respect to the applied field. To obtain the true value of the spin angle from the measurement of the (003) magnetic hematite peak in the polarization analysis experiment we first have to correct the data for imperfect polarization of the neutron beam. Figure 5 shows NSF and SF scans of the (003) structural ilmenite and magnetic hematite peaks before the correction. It can be seen that there is a signal

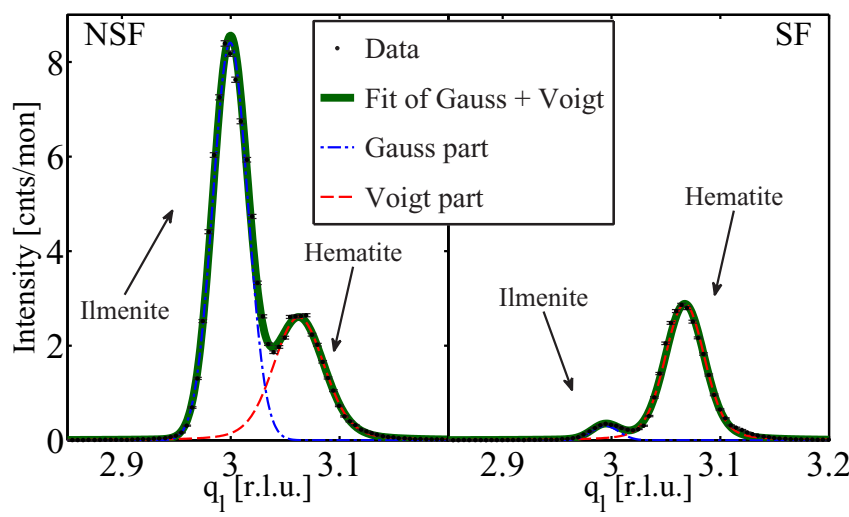

FIG. 5. (Color online) NSF and SF measurement of the (003) structural ilmenite peak and the (003) magnetic hematite peak. This measurement was in an applied field of $0.25 \mathrm{~T}$ and at a temperature of $65 \mathrm{~K}$. This data have not been corrected for imperfect polarization as can be seen from the nonzero SF intensity at the position of the structural ilmenite peak. at the structural peak position even in the SF measurement. A nonmagnetic scattering event cannot change the spin state of the neutron and the nonzero SF intensity on the structural position is caused by the fact that the polarization of the beam is not perfect. The data were corrected for imperfect beam polarization using the following formalism:

$$
p=\frac{n_{\uparrow}-n_{\downarrow}}{n_{\uparrow}+n_{\downarrow}}=\frac{R-1}{R+1}, \quad p_{\uparrow}=\frac{1+p}{2}, \quad p_{\downarrow}=\frac{1-p}{2},
$$

where $p$ is the beam polarization, $n_{\uparrow}$ and $n_{\downarrow}$ are the numbers of neutrons with spin up $(|\uparrow\rangle)$ and down $(|\downarrow\rangle)$, respectively, and $R$ is the so called flipping ratio. The probability of finding a neutron in $|\uparrow\rangle(|\downarrow\rangle)$ is given by $p_{\uparrow}\left(p_{\downarrow}\right)$. The relationship between the true cross sections defined in Eqs. (1) and (2) and the measured intensities $I^{\mathrm{NSF}}$ and $I^{\mathrm{SF}}$ is then

$$
\left(\begin{array}{c}
I^{\mathrm{NSF}} \\
I^{\mathrm{SF}}
\end{array}\right)=\left(\begin{array}{ll}
p_{\uparrow} & p_{\downarrow} \\
p_{\downarrow} & p_{\uparrow}
\end{array}\right)\left(\begin{array}{c}
\sigma^{\mathrm{NSF}} \\
\sigma^{\mathrm{SF}}
\end{array}\right) .
$$

The flipping ratio can be calculated from a measurement of a structural peak $\left(\sigma^{\mathrm{SF}}=0\right)$ :

$$
R=\frac{I_{\text {struct }}^{\mathrm{NSF}}}{I_{\text {struct }}^{\mathrm{SF}}}
$$

and the true cross sections can then be calculated by inverting Eq. (5). Figure 6 shows the data in Fig. 5 after correction for imperfect polarization. For a full treatment of data corrections in a polarization analysis experiment see the excellent review by Wildes [30]. The flipping ratio was obtained from measurements of the structural (003) ilmenite peak for each combination of temperature and applied field. These $R$ values were used to obtain the true SF and NSF cross sections from all measurements.

To confirm the AFM to paramagnetic second order phase transition of ilmenite the $\left(10 \overline{\frac{1}{2}}\right)$ magnetic ilmenite peak was measured and the peak amplitude is displayed as a function of temperature in Fig. 7. The data was fitted to the function

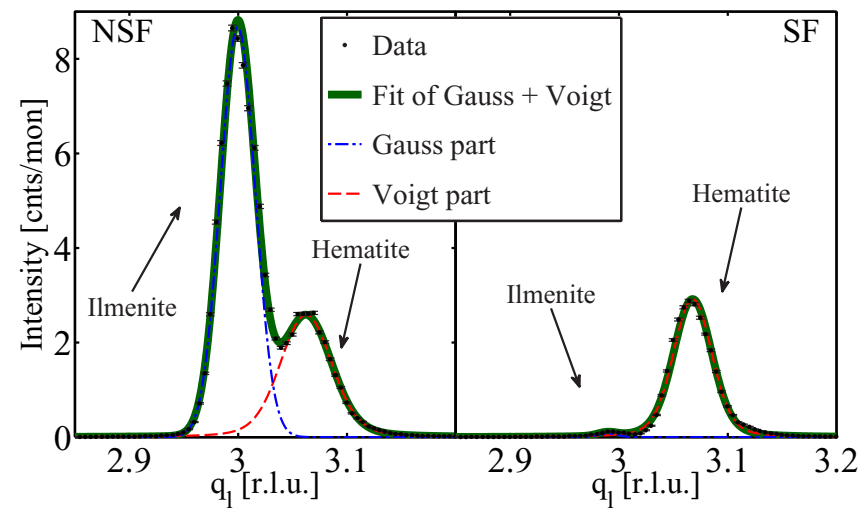

FIG. 6. (Color online) NSF and SF measurement of the (003) structural ilmenite peak and the (003) magnetic hematite peak. This measurement was in an applied field of $0.25 \mathrm{~T}$ and at a temperature of $65 \mathrm{~K}$. This data has been corrected for imperfect polarization with a flipping ratio of $R=43$. The ilmenite reflection is only present in the NSF signal, whereas the hematite peak is present in both the NSF and the SF signal. 


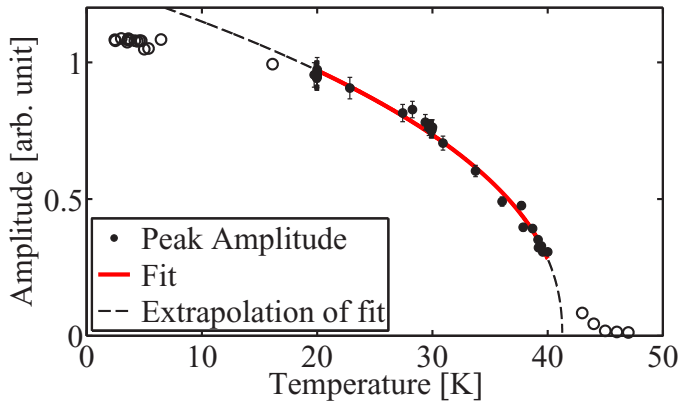

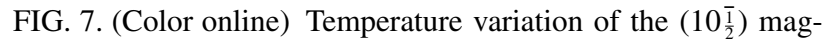
netic ilmenite peak, showing the second order phase transition. Both black and hollow points are peak amplitude measurements, however, the fit is to the black points only, since the power law behavior is only valid within approximately this range.

$A=A_{0}\left(\frac{T_{\mathrm{N}}-T}{T_{\mathrm{N}}}\right)^{2 \beta}$, obtaining $T_{\mathrm{N}}=41.3 \pm 0.2 \mathrm{~K}$ and $\beta=0.22 \pm$ 0.01 . The Néel temperature of $41.3 \mathrm{~K}$ is smaller than the $58 \mathrm{~K}$ usually quoted for ilmenite, which is due to partial substitution of $\mathrm{Mg}^{2+}$ for $\mathrm{Fe}^{2+}$ within the ilmenite lattice, as well as minor solid solution of $\mathrm{Fe}_{2} \mathrm{O}_{3}$.

The intensities of the (003) peaks were obtained through a fit of a Gaussian plus a Voigtian profile to the data (see Fig. 6). The former fits the structural ilmenite peak (bulk material) and the latter fits the magnetic hematite peak (Lorentz broadened peak caused by nanosize effects). For each scan of the (003) peaks the data was corrected in the described way, using the obtained polarization. Figure 8 shows the in-plane spin orientation with respect to the applied field calculated from Eq. (3), which is the main result of our investigations. At all temperatures $\theta$ is close to $45^{\circ}$ in zero field, which is consistent with a nearly random alignment of the hematite magnetic moments within the basal plane. This reflects that the alignment of the hematite moments in zero field due to any remanent magnetization is small and produces a deviation of $\theta$ from $45^{\circ}$ too small to be measured with any significant precision. When the field is applied $\theta$ increases. For the measurements at temperatures of $150 \mathrm{~K}$ and

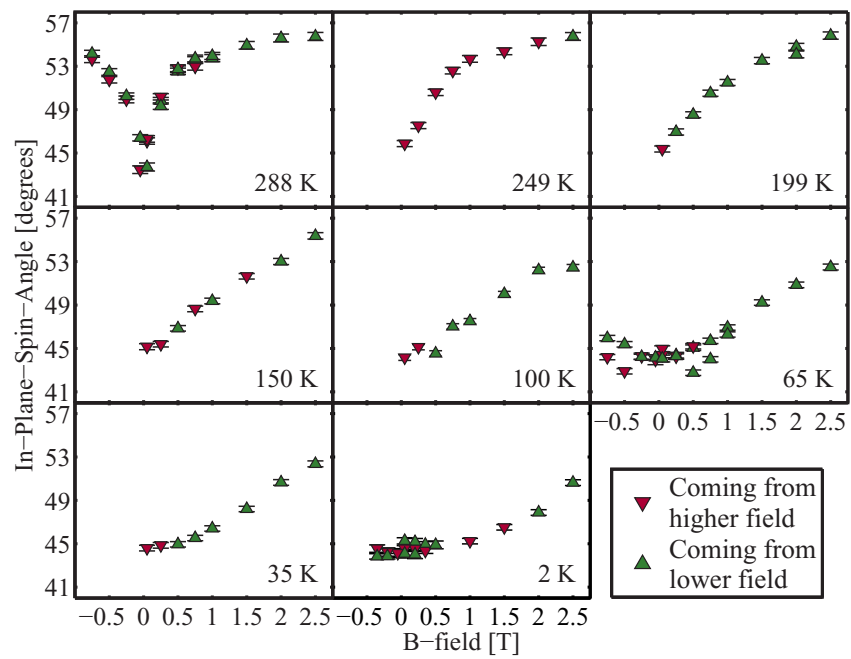

FIG. 8. (Color online) Spin orientation as a function of applied field for different temperatures. The error bars were obtained from Monte Carlo simulations based on the errors on the Voigtian fits. higher, $\theta$ reaches approximately $56^{\circ}$ at the maximum field of $2.5 \mathrm{~T}$, while it only reaches angles of $50^{\circ}-53^{\circ}$ at lower temperatures. At 2, 65, and $288 \mathrm{~K}$ measurements in negative fields of increasing magnitude were performed to investigate the hysteresis of the sample. As can be seen in Fig. 8 no significant hysteresis was observed at 288 and $2 \mathrm{~K}$, while there is a small, but distinct hysteresis feature in the $65 \mathrm{~K}$ data. In the measurement at $288 \mathrm{~K}$ the material is more magnetically soft than at lower temperatures and the magnetization looks to be approaching saturation at approximately $56^{\circ}$. The change in coercivity may be related to increased pinning of $60^{\circ}$ and $120^{\circ}$ domain walls in hematite at low temperatures. There is no significant change in the spin orientation or the susceptibility between 65 and $35 \mathrm{~K}$, indicating that the hematite moments are not strongly coupled to the ilmenite which orders at $41.3 \mathrm{~K}$.

The data in Fig. 8 are insufficient to conclude whether $\theta$ has reached saturation at the applied field of $2.5 \mathrm{~T}$ or not. Therefore the intensity of the (101) magnetic hematite peak was measured with unpolarized neutrons at RITA-II in applied fields up to $11 \mathrm{~T}$. The field was applied in the (003) plane, while observing the (101) peak-the same geometry as in the IN12 experiment. The intensity of the (101) peak is proportional to the square of the projection of the magnetic moment perpendicular to the (101) scattering vector and its response to a magnetic field can therefore reveal the saturation field of the hematite moments. The (101) intensity was measured at 2 and $150 \mathrm{~K}$ and thus above and below the Néel temperature of ilmenite. The results are displayed in Fig. 9. In the $150 \mathrm{~K}$ data the (101) intensity decreases from $0 \mathrm{~T}$ to approximately $2.5 \mathrm{~T}$ and then increases to $11 \mathrm{~T}$. This is consistent with a saturation of the (net) hematite moments followed by a rotation of the

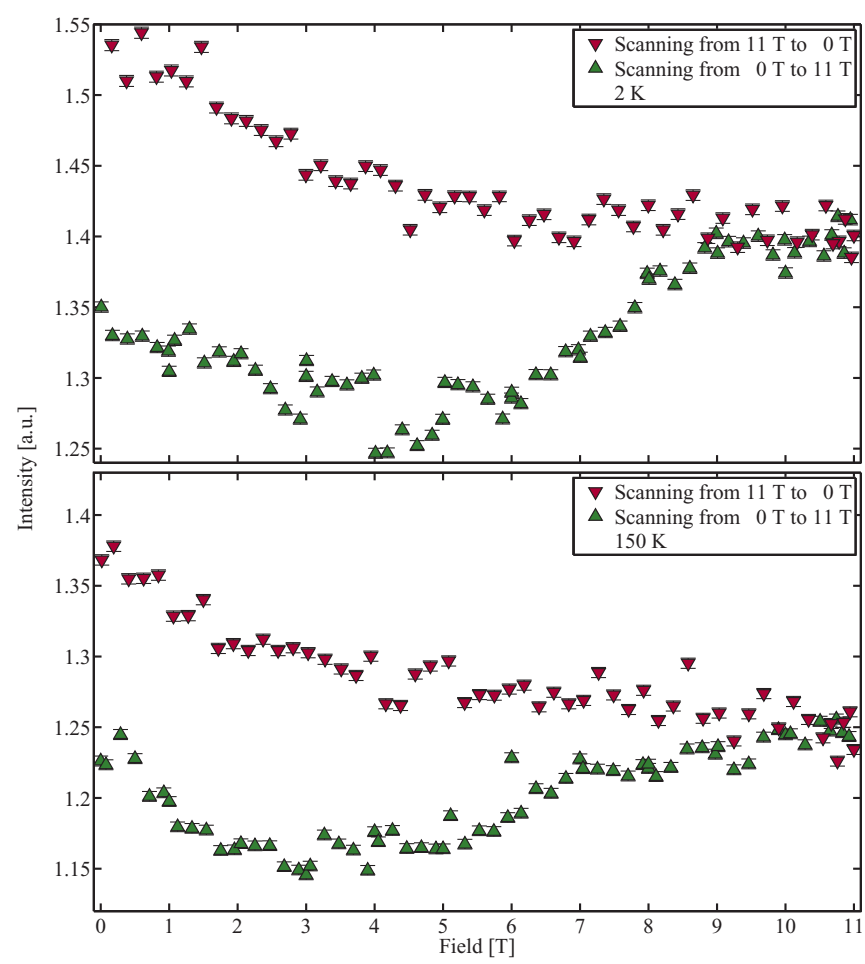

FIG. 9. (Color online) Unpolarized nuclear diffraction measurement at 2 and $150 \mathrm{~K}$ of the (101) peak, for applied fields between 0 and $11 \mathrm{~T}$. 
AFM sublattices away from antiparallel (AFM susceptibility). When the field is decreased to zero again there is a significant hysteresis. The picture is very much the same in the $2 \mathrm{~K}$ data, except that the saturation point is not reached before approximately $5 \mathrm{~T}$. This confirms that for the data in Fig. 8 the value of $\theta$ reaches saturation in the measurements at temperatures above $150 \mathrm{~K}$, whereas it is unsaturated at lower temperatures.

\section{DISCUSSION}

The strong NRM in natural hemo-ilmenite samples is believed to be caused by uncompensated magnetic moments in contact layers between exsolution lamellae (lamellar magnetism). The magnetization would then be a combination of these lamellar moments and the CAF moments. In a saturating magnetic field the net moment is expected to align parallel to the field (see Fig. 2). It is important to note that the measured angle $\theta$ is not the orientation of the net moment, but the average spin orientation. In a model with CAF moments only, the spins would be expected to align nearly perpendicular to the applied field $\left(\theta=90^{\circ}\right)$. At saturation the only deviation from perpendicular will be the insignificant canting angle. For lamellar moments alone the moments would align themselves parallel to the applied field $\left(\theta=0^{\circ}\right)$, whereas a combined lamellar and CAF moment would align at an intermediate angle. The spin angles measured at temperatures above $150 \mathrm{~K}$ are saturated at the $2.5 \mathrm{~T}$ field, as confirmed by the high-field measurements at RITA-II displayed in Fig. 9. The saturation value of the angle is approximately $56^{\circ}$ (Fig. 8). The measured spin angle is an average over all of the hematite spins in the sample and the result is thus consistent with a model with a combination of CAF moments aligning perpendicular to the field and lamellar moments aligning parallel to the field. If all the spins were aligned either perpendicular or parallel to the applied field the $56^{\circ}$ would correspond to approximately $62 \%$ of the moments being CAF and $38 \%$ lamellar moments. The ratio of lamellar to CAF moments will in general depend on the relative surface area of the lamellae and it is likely that the lamellar contribution will be larger in samples with more fine scale lamellae compared to the rather coarse microstructure in the sample investigated here. While this picture is certainly too naive, the $56^{\circ}$ angle is evidence that a significant proportion of the spins are aligned parallel to the field, consistent with a model with uncompensated moments in contact layers between lamellae as important for producing the large NRM. We note that our experiment cannot determine whether the magnetic moments here termed lamellar moments are uncompensated magnetic layers exactly as described in the lamellar magnetism hypothesis $[4,20,23,24]$ or perhaps randomly placed uncompensated magnetic moments on the interfaces between the two phases like the uncompensated magnetic moment known to produce a significant net magnetization in antiferromagnetic nanoparticles of, e.g., $\mathrm{NiO}$ [31,32]. The hematite moments respond to the magnetic field in much the same way above and below the ordering temperature of ilmenite indicating that the effect of any coupling between the lamellar moments and the ilmenite moments is relatively weak in this particular sample.

\section{CONCLUSION}

We have reported results from a neutron diffraction experiment with uniaxial polarization analysis performed on a natural hemo-ilmenite sample with a fine exsolution structure. Measurements of the $\left(10 \frac{\overline{1}}{2}\right)$ ilmenite peak confirms that the ilmenite undergoes a second order phase transition from paramagnetic to antiferromagnetic at a Néel temperature of $41.3 \mathrm{~K}$. Measurements of the (003) hematite peak show that the hematite spin angle is close to $45^{\circ}$ in zero applied field independent of temperature. This corresponds to a close to random alignment of the hematite moments. When a magnetic field is applied, the in-plane hematite moments rotate away from the field. At temperatures from 150 to $288 \mathrm{~K}$ the moment saturates in the maximum applied field of $2.5 \mathrm{~T}$, making an angle of about $56^{\circ}$ to the field. At lower temperatures the moment is not saturated in the $2.5 \mathrm{~T}$ field. The saturation angle of $56^{\circ}$ with respect to the applied field strongly supports the hypothesis of lamellar magnetism as part of the explanation for the natural remanent magnetism in natural hemo-ilmenite. Our data rule out a model with either lamellar moments or CAF moments as the sole explanation for the NRM and shows that the magnetization is a sum of contributions of similar size of uncompensated moments in contact layers and CAF moments.

\section{ACKNOWLEDGMENTS}

The authors would like to thank the late J. M. Knudsen and the late R. B. Hargraves for inspiring discussions and for suggesting this puzzling nanomagnetism topic for neutron studies. The research leading to these results has received funding from the European Research Council under the European Union's Seventh Framework Programme (FP/20072013), ERC Grant Agreement No. 320750, and the Natural Environment Research Council Grant NE/B501339/1. This work was supported by the Danish Agency of Science and Innovation through DANSCATT.
[1] S. A. McEnroe, R. J. Harrison, M. J. Jackson, A. M. Hirt, P. Robinson, F. Langenhorst, F. Heidelbach, T. Kasama, A. Putnis, L. L. Brown, and U. Golla-Schindler, J. Phys.: Conf. Ser. 17, 154 (2005).

[2] S. A. McEnroe, P. Robinson, and P. T. Panish, Am. Mineral. 86, 1447 (2001).

[3] S. A. McEnroe, J. R. Skilbrei, P. Robinson, F. Heidelbach, and F. Langenhorst, Geophys. Res. Lett. 31, L19601 (2004).
[4] P. Robinson, R. J. Harrison, S. A. McEnroe, and R. B. Hargraves, Nature (London) 418, 517 (2002).

[5] Y. Ishikawa and S.-I. Akimoto, J. Phys. Soc. Jpn. 12, 1083 (1957).

[6] Y. Ishikawa, J. Phys. Soc. Jpn. 13, 37 (1958).

[7] W. H. Butler, A. Bandyopadhyay, and R. Srinivasan, J. Appl. Phys. 93, 7882 (2003).

[8] H. Hojo, K. Fujita, K. Tanaka, and K. Hirao, Appl. Phys. Lett. 89, 082509 (2006). 
[9] T. Fujii, M. Kayano, Y. Takada, M. Nakanishi, and J. Takada, Solid State Ionics 172, 289 (2004).

[10] H. Kato, M. Yamada, H. Yamauchi, H. Hiroyoshi, H. Takei, and H. Watanabe, J. Phys. Soc. Jpn. 51, 1769 (1982).

[11] A. H. Morrish, Canted Antiferromagnetism: Hematite (World Scientific, Singapore, 1994).

[12] F. J. Morin, Phys. Rev. 78, 819 (1950).

[13] P. J. Besser, A. H. Morrish, and C. W. Searle, Phys. Rev. 153, 632 (1967).

[14] R. M. Cornell and U. Schwertmann, The Iron Oxides (Wiley, Weinheim, 2003).

[15] D. H. Lindsley, Carnegie Inst. Washington Year Book 64, 144 (1965).

[16] U. Schärer, E. Wilmart, and J. C. Duchesne, Earth Planet. Sci. Lett. 139, 335 (1996).

[17] S. A. McEnroe, R. J. Harrison, P. Robinson, and F. Langenhorst, Geophys. J. Int. 151, 890 (2002).

[18] R. J. Harrison, S. A. McEnroe, P. Robinson, and C. J. Howard, Am. Mineral. 95, 974 (2010).

[19] P. Robinson, F. Heidelbach, A. M. Hirt, S. A. McEnroe, and L. L. Brown, Geophys. J. Int. 165, 17 (2006).

[20] P. Robinson, R. J. Harrison, S. A. McEnroe, and R. B. Hargraves, Am. Mineral. 89, 725 (2004).
[21] S. A. McEnroe, P. Robinson, F. Langenhorst, C. Frandsen, M. P. Terry, and T. Boffa Ballaran, J. Geophys. Res. 112, B10103 (2007).

[22] P. Robinson, K. Fabian, S. A. McEnroe, and F. Heidelbach, Geophys. J. Int. 192, 514 (2013).

[23] S. A. McEnroe, B. Carter-Stiglitz, R. J. Harrison, P. Robinson, K. Fabian, and C. McCammon, Nat. Nanotechnol. 2, 631 (2007).

[24] K. Fabian, S. A. McEnroe, P. Robinson, and V. P. Shcherbakov, Earth Planet. Sci. Lett. 268, 339 (2008).

[25] J. C. Duchesne, Mineral. Deposita 34, 182 (1999).

[26] P. Böni and P. Keller, PSI Proc. 2, 35 (1996).

[27] E. Brok, Master thesis, University of Copenhagen, 2009.

[28] K. Lefmann, C. Niedermayer, A. B. Abrahamsen, C. Bahl, N. Christensen, H. Jacobsen, T. Larsen, P. Häfliger, U. Filges, and H. Rønnow, Phys. B: Condens. Matter 385-386, 1083 (2006).

[29] R. Moon, T. Riste, and W. Koehler, Phys. Rev. 181, 920 (1969).

[30] A. R. Wildes, Neutron News 17, 17 (2006).

[31] J. T. Richardson, D. I. Yiagas, B. Turk, K. Forster, and M. V. Twigg, J. Appl. Phys. 70, 6977 (1991).

[32] C. R. H. Bahl, M. F. Hansen, T. Pedersen, S. Saadi, K. H. Nielsen, B. Lebech, and S. Mørup, J. Phys.: Condens. Matter 18, 4161 (2006). 